A CASE

OF

\title{
ANEURISM OF THE ARCH OF THE AORTA
}

INVOLVING THE

\section{INNOMINATE ARTERY :}

WITH REMARKS ON THE DISTAL LIGATURE.

BY

HENRY MORRIS, M.A., F.R.C.S.,

SURGEON TO, $\triangle N D$ LECTURER ON SURGERY $\triangle T$, THE MIDDLESEX HOSPITAL.

Received February 6th-Read February 13th, 1883.

THE following case presents features which seemed to encourage the operation of ligature of the common carotid. It also shows the untrustworthiness of any conclusions, either as to the operation to adopt, or as to the anatomical relations of the aneurism, drawn from the fact that distal pressure on one of the large arteries in connection with the tumour diminishes the force of the aneurismal pulsation.

Sarah B-, a married woman, æt. 43 , a "farm labourer," was admitted into the Middlesex. Hospital on September 7th, 1882. 
History.-Six months ago she noticed a severe gnawing pain across the front of the sternum which she attributed to indigestion. Three months ago she began to feel after exertion "a great throbbing and beating" just above the inner end of the right collar bone, which became worse by degrees. Quite lately she had felt pain shooting up the right side of the neck to the ear, and through the thick part of the shoulder. She had no cough, no dysphagia, had never had syphilis, and her general health had always been good.

State on admission.-A dark, fresh-complexioned, spare woman, with an expansile pulsating tumour plainly seen as well as felt above the right sterno-clavicular joint and inner fifth of the right clavicle. The upper limit of pulsation was fully an inch and a quarter above the upper border of the clavicle; the inner limit reached the median line of the neck, the outer corresponded to the outer border of the sterno-mastoid. Pulsation was also felt in the first and second intercostal spaces for at least an inch outwards from the right edge of the sternum. The inner half of the clavicle rose and fell with the aneurism, and its sternal extremity looked partially dislocated forwards. There was a loud bruit over the whole of the tumour. The external jugular and other veins on the right side of the neck and upper part of the chest were dilated. There was no irregularity of the pupils, no appreciable difference in the pulse at the wrists (or, if any, it was the left that was slightly weaker), nor in the facials, but the right temporal was more feeble than the left. By compressing the trunk of the right common carotid a distinct diminution in the force and fulness of the pulsation in the aneurism was produced-this was quite marked and readily appreciated by the eye and finger. Compression on the third part of the subclavian caused no difference in the aneurism. The tissues over the cervical part of the tumour were very thin. The pulse was small and weak, 116 when sitting and 104 when lying down.

I resolved on trying Tufnell's treatment, and ordered one 
grain of acetate of lead in a pill three times a day. On this treatment she improved, her pulse dropped, so that on the 9 th of September it was 88 , on the 11 th 80 , on the 13th 70 , and from this time it continued to be between 70 and 76 ; it was also regular, compressible, and small. The aneurism also improved. By September 19th the clavicle was no longer raised by the pulsation, and the appearance of luxation at the sterno-clavicular joint had gone.

So she continued till October 4th, when she complained of giddiness and pain in the head; the aneurism pulsated more than it had done for many days previously, and the inner end of the clavicle again rose and fell with the pulsation. Between this date and October 20th the tumour became softer and extended further to the left, reaching beneath the border of the left sterno-mastoid. Its impulse was more plainly felt in the first intercostal space than it had been, and there was some slight bulging of the upper space and first two cartilages of the right side.

On October 20th, after consultation with Dr. Powell, iodide of potassium in twenty-grain doses three times a day was ordered, and the pills were of course discontinued. The diet was to remain the same. Up to this time there had been no symptom whatever of plumbism, though the blue line on the gums had been looked for several times; but, after taking the iodide of potassium for nine days she developed the blue line, experienced colicky pains in the abdomen, loss of appetite and sickness. The iodide was therefore discontinued on 31st October, and the restricted diet on November 2nd.

By November 14th all symptoms of plumbism had disappeared except the blue line, and this was fast fading. The condition of the aneurism was about the same. She was allowed to sit up to-day for the first time since treatment was commenced. It was now noticed that the pulsation along the carotid artery was feebler, but distal compression still produced an appreciable effect on the aneurism.

On November 25th, at 2.30 p.m., ether was administered 
and the operation of ligaturing the carotid was commenced. After the usual incision for applying the ligature on the level of the omohyoid, what looked like the sheath of the cervical vessels was opened and a feebly pulsating vessel smaller and thinner than a normal common carotid, but with none of the appearance of a large vein was brought into view. No other vessel could be found. The pneumogastric nerve lay to the inner side of the vessel upon a quantity of sclerosed fibrous tissue. Whilst the vessel was being held aside for the purpose of examining this tissue, it was injured and a rush of venous blood flooded the wound, flowing as from a vein. Torsion forceps secured the opening and controlled the hæmorrhage, and a further examination of the parts was made without discovering the artery. I then dissected the wounded vein downwards towards the aneurism, and found it pulsating forcibly near the sac. I tied the vein above and below the wound in it with ox aorta ligature, very kindly supplied to me by Mr. Barwell. On tightening the lower ligature the aneurismal pulsation diminished somewhat and the knot of the ligature moved forcibly with each beat of the aneurism.

For several hours after the operation the patient lay in a semi-conscious state, but nothing requiring notice occurred after she rallied until the morning of the $27 \mathrm{th}$, when the right side of the face was looking waxy and odematous, and she complained of pain over the lower part of the right side of the neek. There was considerable cdema and redness between the clavicle and trapezius, but the wound looked united and the tissues immediately around it were quite natural in appearance. During the next two days the cedema and pain increased. Subsequently matter formed and burrowed inwards to the wound and downwards to the upper edge of the great pectoral muscle ; incisions were made, and the wound opened up, and a quantity of pus was discharged. There were no rigors, and very slight constitutional disturbance until two days before death, when the respirations and pulse were rapid, the 
temperature rose to $103 \cdot 6^{\circ}$, suppuration became very free, the aneurismal pulsation increased, and the patient rapidly sank and died of asthenia on December 9th.

A post-mortem examination was made on December 11th by Dr. Fowler, and the aneurism, aorta, and large vessels of the neck were subsequently carefully dissected by Mr. Sutton.

The following notes are extracted from the post-mortem report :

There were the evidences of diffused suppuration between the skin and platysma, and beneath the platysma, in the right posterior triangle of the neck. The suppurating areas communicated with the exterior through three incisions, as well as through the operation wound. The aorta, from the semilunar valves to about half an inch beyond the origin of the left subclavian, was dilated, and measured (after being in spirit several days) $3 \frac{5}{3}$ inches in circumference at the junction of the ascending and transverse portions of the arch. The tunica intima was much thickened, and dotted with patches of atheroma.

At the point of origin of the innominate artery and extending a little to its right side was a circular opening in the aorta $1 \frac{3}{4}$ inches in circumference, and therefore larger than the normal orifice of the innominate. This opening led into a sacculated aneurism of the arch of the aorta, as well as into the innominate vessel, the lower end of the right wall of which was deficient for half an inch and presented a crescentic, rounded, thickened edge:

The sac of the aneurism was oval, and measured (after contraction in spirit) about $1 \frac{3}{4}$ inches vertically, by 1 across; it contained a small quantity of laminated clot about its base, elsewhere there was little or none. The postero-internal wall of the sac was formed partly by the innominate artery, the external coat of which had been raised upwards by the growth of the aneurism for a distance of $\frac{5}{8}$ of an inch ; thus, on looking into the aneurism, a concave triangular shaped area was seen at this point where the wall of the artery was exposed.

voL. LXVI. 
The innominate vessel from origin to bifurcation, as well as the commencements of the right subclavian and common carotid arteries, were behind the aneurism. Both subclavian and common carotid were considerably dilated at their origin (the subclavian for the first inch of its course), and their coats, as well as those of the innominate, were much thickened.

The common carotid was firmly bound down by a dense, thick, layer of sclerosed connective tissue, which stretched from the sac of the aneurism as high as the upper border of the larynx. The vessel was thus completely flattened and shut out of view, and it was only after several minutes of difficult dissection, with the parts all laid bare, that it could be found. At a distance of $1 \frac{3}{8}$ inch from its origin the artery was completely occluded, all attempts to pass the finest probe through it being ineffective. This occlusion continued for $\frac{1}{8}$ inch, when the vessel became again pervious. Just above the occlusion the carotid was somewhat dilated, as it was also near its bifurcation.

The right internal jugular vein bore two ligatures about an inch apart, opposite the larynx; the lower ligature moved up and down upon the collapsed vein like a ring; the upper one became detached during dissection. The lower part of the vein was intimately incorporated with the right wall of the aneurism, but there was a free channel through it into the right innominate vein. Decolorised clot extended from the lower ligature into the portion of the jugular adherent to the sac. No communication existed between vein and aneurism. The left innominate vein was completely occluded; so that after the right internal jugular was tied, all blood from the head and neck must have returned by the right external jugular and small anastomotic branches. The pneumogastric nerve was closely adherent to the back of the aneurism on its right side.

The other viscera were examined, but require no notice.

Remarks. - The case illustrates the extreme difficulty of diagnosing some forms of aortic aneurism, from aneurism 
of the innominate. No one who examined this woman questioned that the aneurism was innominate, and some very capable diagnosticians considered it to be a simple sacculated aneurism of that vessel. Even after dissection it was impossible to make out its true character and connection until the sac had been laid freely open in front, and the innominate artery behind.

The situation and outline of the tumour; the pain in the shoulder and over the right side of the head and neck; the shooting character of the pain, and the venous congestion of the right side of the neck led to the diagnosis of innominate aneurism. The origin of the disease from the aorta might have been suspected if more weight had been given to the severe guawing pain across the front of the chest suffered at the outset; to the dilated veins on the right side of the upper part of the chest ; to the equality of the radial pulses ; to the absence of any cough, dyspncea, and throat dryness, of any deflection of the trachea, of any numbness or loss of power in the right arm, - such frequent symptoms in innominate aneurism; and to the fact that aortic aneurisms cause tumour in the neck. When, however, characteristic symptoms are blended as they were in this case, a precise diagnosis is not possible.

The marked effect on the aneurism of pressure on the common carotid, and the negative effect of compressing the third part of the subclavian are features of great importance. Possibly it was in part due to the closer and greater length of contact of the carotid with the sac; but after events lead me to think that the chief effect is probably attributable to the simultaneous compression of the pneumogastric trunk. Sir William Fergusson supposed that the pressure caused its effect by its influence on the brain and on the action of the heart, because similar symptoms were produced in his case of innominate aneurism by pressing on the left carotid. ${ }^{1}$

1 Sir Wm. Fergusson, 'Lond. and Edinb. Monthly Journ.,' Nov., 1841, p. 793. 
The great diminution in the pulsation of the aneurism by distal pressure on the common carotid decided me to tie that vessel alone, with the hope that laminated clot would extend from its orifice into the sac. The case, however, like others, shows how little value can be attached to a difference in the effect of pressure on the carotid, and on the subclavian. ${ }^{1}$ Sir William Fergusson was led to tie the carotid alone, yet his case turned out to be an aneurism of the innominate involving the subclavian. In Mr. Holmes's ${ }^{2}$ case of aortic aneurism involving the innominate, pressure on the subclavian produced the greater effect, and on this account, as he mistrusts the ligature of the third part of the subclavian alone, he simultaneously ligatured it and the carotid; yet after death " the arteries opening out of the tumour seemed healthy," and there was no extension of clot into the aneurism from either of them. The anatomical relation of the subclavian artery to the aneurism in my case does not lead me to suppose that the aneurism would have been cured, or even in any degree improved, if the subclavian, even in the first part of its course as well as the carotid, had been successfully ligatured.

Nature sometimes performs for us an experiment, equivalent to the double ligature, without effecting thereby the cure of the aneurism. Thus Dr. Douglas Powell has

1 With reference to the effects of pressure on the carotid artery, my atten. tion has been drawn by Mr. Roger Williams to the following interesting remarks by Dr. M. Moir in his 'Outlines of the Ancient History of Medicine' (1831, p. 138). Speaking of Rufus, the Ephesian, one of Galen's predecessors, who died in the reign of Trajan, Dr. Moir says, "In his anatomical treatise he mentions the recurrent nerve, which had then been newly discovered; and, in the same, makes the remark that pressure on the carotid arteries had been observed to take away the voice. He points out, however, the true cause of this fact, by showing that it does not arise from pressure on the arteries, but on the nerves, which are contiguous to them." Portal ("Histoire de l'Ana. tomie,' tome i, p. 74) has also quoted this same observation of Rufus.

See also Mr. Savory on the influence of pressure on the pneumogastric nerve in the neck ('Lancet,' June 9, 1883).

2 'Lancet,' 1872. Holmes Lectures on A neurism. 
recorded a case in the twentieth volume of the Pathological Society's 'Transactions,' p. 118, of an aneurism of the arch of the aorta implicating the whole length of the innominate, in which, after death, it was ascertained that both the carotid and origin of the subclavian were quite obliterated, and yet scarcely any laminated clot existed in the aneurism. Dr. Powell's case was peculiarly like mine ; and in his, as in mine, it appeared at first sight, on dissection, that the aneurism was connected with "an aorta from which the subclavian and right common carotid were given off as separate trunks."

It does not seem from the cases tabulated by authors and those more recently made public, that the distal ligature affords much encouragement to the surgeon in innominate aneurism. I do not gather from the records of these cases that there is a single instance of complete cure after ligature of the subclavian alone; or ligature of both vessels simultaneously or consecutively; and only one case, namely, Evan's, after ligature of the carotid alone. The great majority of the operations have been complete failures; though a few cases have been temporarily benefited. Bryant's, Wardrop's, and Broca's cases, in which the subclavian only was tied, improved, and the patients lived three years, two years and a quarter, and seven months respectively. Of the cases in which the carotid alone was tied, Morrison's and Mott's were relieved, and the patients lived twenty months and seven months respectively, whilst one of Pirogoff's cases recovered from the operation but was not cured of his disease. Again, of the cases in which both vessels were ligatured, all died within periods varying from several hours to four months (Fearn's), with the exception of Langley Browne's, which was probably of traumatic origin, and Barwell's three cases all of which were much relieved by the operation, though all have since died.

1 'Med.-Chir. Trans.,' vol. lxv, p. 21. Browne's case lived about one year. No post-mortem. 
In estimating the value of the distal ligature, too much importance has perhaps been attached to cases such as Fearn's, Morrison's, and others, in which, after death, the aneurism has been found filled with clot. ${ }^{1}$ As distinct from the effects of ligature, and with regard to the formation of clot, some importance, ought to be given to the condition of the blood and general state of circulation brought about by the fatal disease, as well as to the slow approach of death; circumstances which in themselves favour, in no small degree, the deposition of laminated fibrine.

As regards aortic aneurisms; I think it may be questioned whether Mr. Heath's well-known case of double distal ligature, which hitherto has proved quite exceptional in its success, has not placed Wardrop's operation in a light altogether more favourable than subsequent experience warrants. This opinion, I venture to think, is not invalidated by such cases of double distal ligature for aortic disease as Barwell's (two), and Lediard's, whose patients died at the end of seventeen, fifteen, and nine months after the operation respectively; or of Wyeth's, whose patient recovered from the operation and was alive three months after it, but with the aneurism still uncured.

Such cases as Rigen's, Tillanus's, Holme's's, and Heath's, of ligature of the left common carotid for aortic aneurism ought not, I would submit, to be weighed in the same balance as the operation on the right side. The operation on the left ressel, in suitable cases, gives the patient a much better chance of cure, because the ligature has nearly all the advantages, without the risk, of ligature of the innominate for aneurism of the right part of the arch. It is Brasdor's operation; whilst there is only half the risk and a third more advantage than in the double distal ligature.

1 That laminated clot is not tantamount to cure, but is only a step in the process of cure, of an aneurism is not sufficiently appreciated : but this is proved by many cases on record, and is to be seen from time to time in the post-mortem room, in instances in which, though the aneurism is almost if noi quite full of laminated clot, the patients, nevertheless, have died from hæmorrhage owing to the giving way of the sac. 
It will, perhaps, be admitted that when the tumour is not limited to the innominate and its derivatives, but is partly or entirely aortic, the chances of complete obliteration of the sac by the double ligature are much diminished. If the enormous difficulty of diagnosis, the great risks of the operation, the possibility of spontaneous improvement, if not of cure, and of palliation by rest and diet and also the fatal results of recorded operations, be taken into due consideration, it seems that the distal ligature on the right side should be limited to desperate cases, and then performed only with the expectation of relief, not of cure: and that as ligature of the carotid has appeared to take a larger share in the consolidation of the aneurism, its effects should be tried before the patient is submitted to the further risk of the operation on the subclavian.

The pulsation in the internal jugular vein due to its connection with the sac of the aneurism is a point of some clinical importance. Though the pulsation along the course of the carotid was for some time before the operation observed to be feebler than at first, yet, as a pulse was perceived, as it was supposed, in the vessel, and as compression along the course of the carotid diminished the aneurismal pulsation and further as constitutional treatment had not succeeded, ligation of the carotid seemed to be indicated. Occlusion of the artery had been accomplished, as it proved, long before the operation was undertaken, but no good came of this spontaneous occlusion; the aneurism, on the contrary, continued to enlarge after the carotid was plugged. Probably the date at which the artery was blocked was between the 4th and 20th of October; certainly the plug had existed a long time before death.

The development of lead-poisoning by the administration of iodide of potassium is noteworthy, and tends to confirm the views expressed by Dr. Fagge in a paper in the fifty-seventh volume of the 'Med.-Chir. Transactions,' except, however, in this particular : that the blue line had not existed during the time the lead pills were taken, 
and appeared only after the iodide of potassium had been commenced.

The condition of the ligatures at the time of death was to all appearances the same as at the time of their application. The specimens of ox-aorta used were found to be brittle as well as elastic, but still they were capable of being tied tightly enough to accomplish their purpose.

I am indebted to Mr. Roger Williams for the following account of the states of the ligatures after the patient's death:

"The noose of one of the ligatures was found to be loose on the included vessel, which had shrunk within it. There were no adhesions between it and the adjacent structures. The knot held as firmly as it had been tied at the operation. To unfasten it was easy, as the several parts in contact were not firmly adherent. After removal, the ligature had a soapy feel; but otherwise it presented no obvious alteration.

"Microscopical examination showed the whole thickness to be infiltrated with granular leucocytes. These were for the most part arranged in bead-like chains between the fibre bundles of the tissue; the latter were evidently in a state of commencing disintegration, presenting where in contact with the chains of leucocytes minute crenated erosions; moreover, these bundles also showed signs of granular degeneration, and in many parts of the specimens they were splintered into short fragments, and in a few they were quite destroyed. A small number of giant cells were detected in the tissue, in one of them I counted thirteen nuclei."

To summarise : the case shows (1) the impossibility of diagnosing with accuracy some forms of aortic from innominate aneurism; (2) the untrustworthiness of any conclusion drawn from the effects upon the aneurism of distal compression of the large arteries; (3) that a very appreciable amount of pulsation may be felt in the jugular vein when it has become adherent to the sac of the aneurism, 
and that this may lead to error if the carotid has become occluded; (4) that the anatomical position of some aortic aneurisms is such that they are not likely to be cured by the occlusion of both subclavian and carotid arteries; and (5) that, as Dr. Fagge has pointed out, iodide of potassium is capable of developing plumbism in persons in whose system the lead is lying inactive. 


\section{DESCRIPTION OF PLATES II AND III.}

Aneurism of the arch of the aorta involving the innominate artery (HENRY MorRIs, M.A., F.R.C.S.).

Platr II.-Aneurism of the arch of the aorta involving the innominate artery. Anterior view.

A. Sac of aneurism stretched open.

B. Superior vena cava.

C. Right subclavian artery.

D. Right subclavian vein.

E. Right common carotid artery.

F. Internal jugular vein, knot of ligature in situ.

G. Clot in internal jugular below point of ligature, and in the part of the vein adherent to the sac of the aneurism.

H. Left common carotid.

I. Left subclavian artery.

J. Pulmonary artery.

K. Right wall of innominate artery forming part of wall of aneurism.

L. Semilunar margin of right wall of innominate.

M. Small aneurismal pouch on posterior and left side of innominate.

$\mathrm{N}$. Left innominate vein thrown over to right side.

Puatr III.-Ditto. Posterior view.

A. Innominate artery laid open behind and exposing the two ponch-like dilatations in its wall, and the rods passing into the carotid and subclavian arteries.

B. Superior vena cava.

C. Subclavian artery.

D. Right vertebral artery.

E. Dilated origin of right common carotid.

F. Knot of ligature on internal jugular vein.

G. Internal jugular vein laid open just below where it leaves the wall of the aneurism, and as it joins right suhclavian vein.

H. Left carotid.

I. Left subclavian.

J. Right pulmonary artery.

K. Opening from innominate into sac of aneurism. 


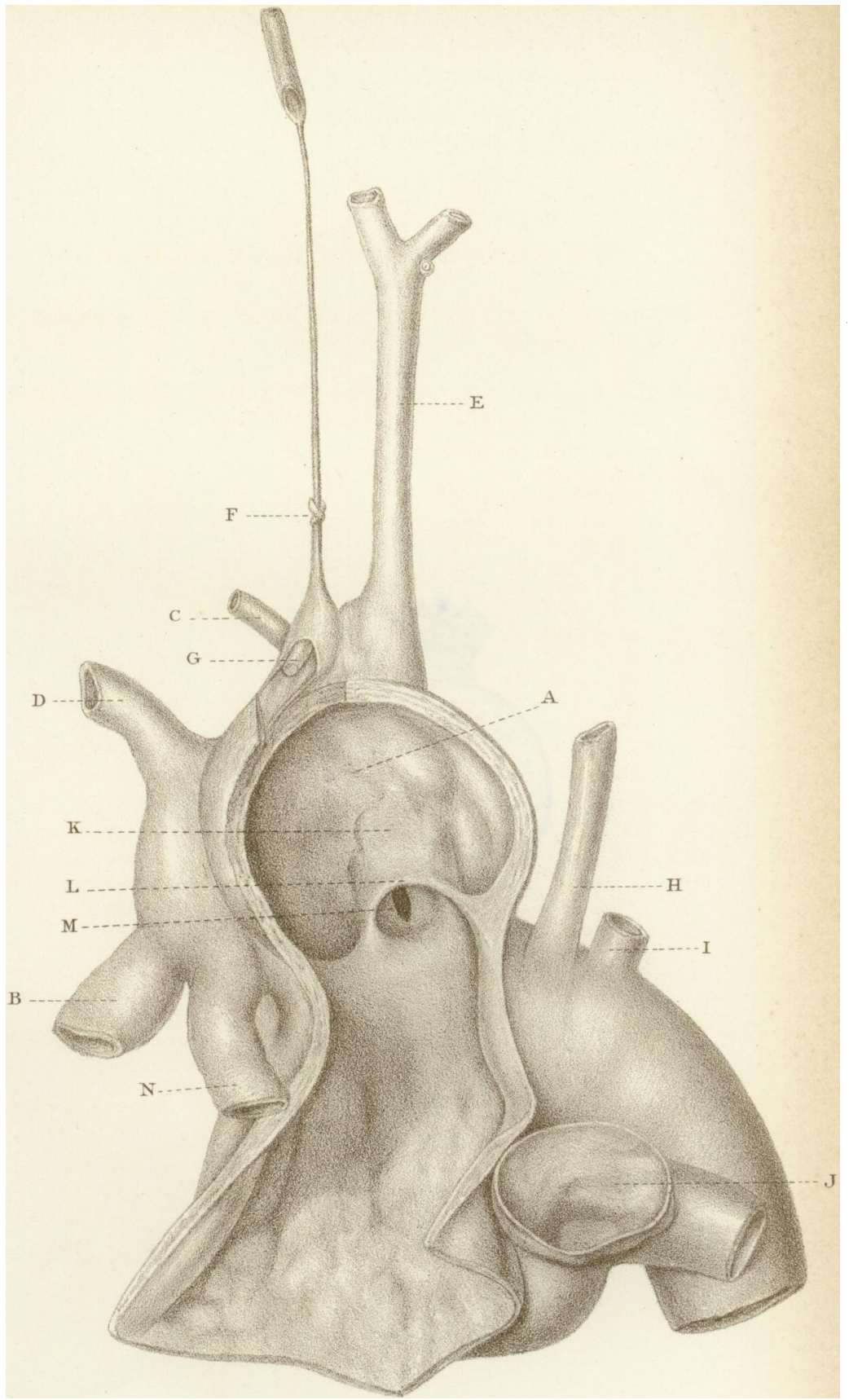


Plate III.

Med.Chir.Trans.Vol. LXVI.

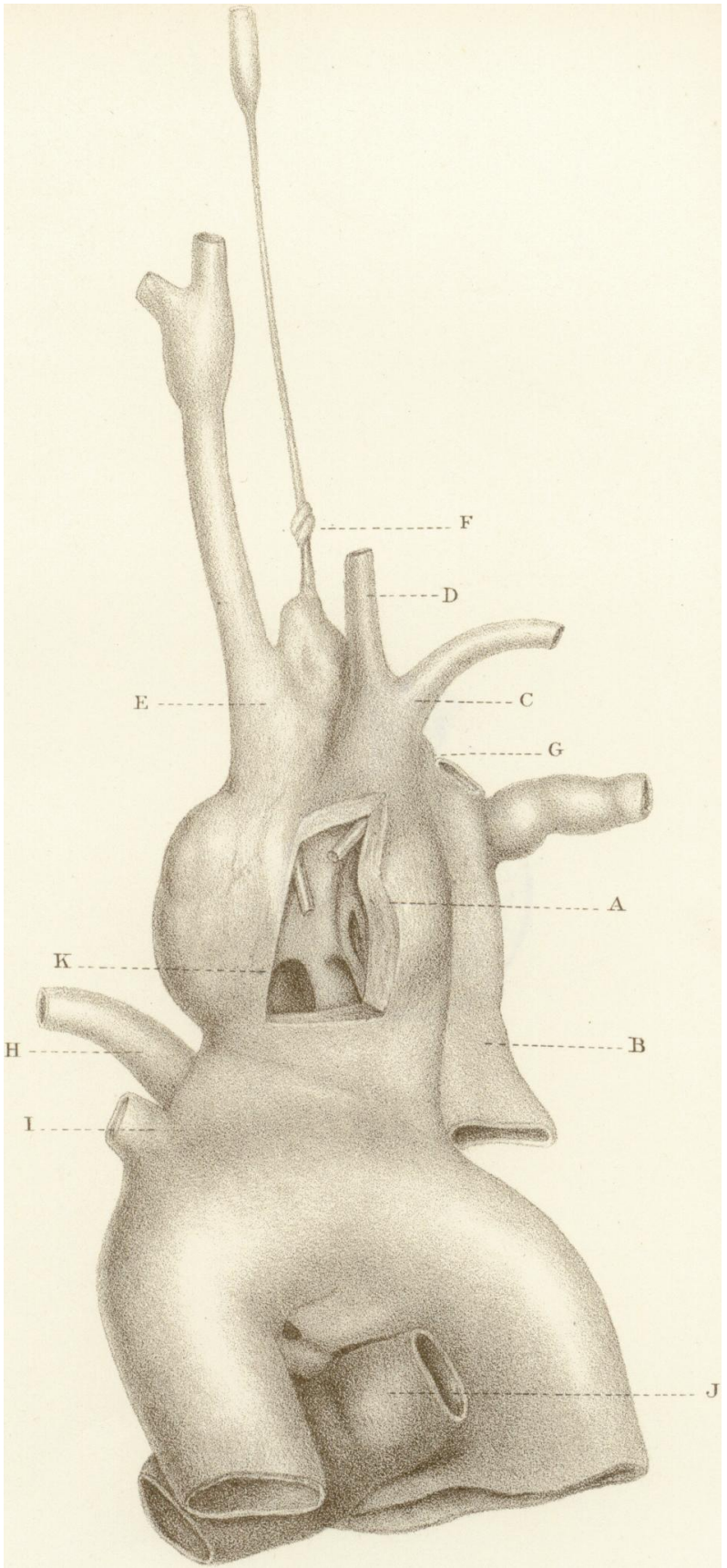

